\title{
Application of MTR soft-decision decoding in multiple-head magnetic recording systems
}

\author{
NIKOLA DJURIC and MIROSLAV DESPOTOVIC \\ Faculty of Technical sciences, University of Novi Sad, Trg D. Obradovic 6, 21000 \\ Novi Sad, Serbia \\ e-mail: ndjuric@uns.ns.ac.yu
}

MS received 25 August 2007; revised 27 January 2009

\begin{abstract}
Application of a simple approach for the soft-decision decoding of Maximum-Transition-Run (MTR) codes has been presented in this paper. A lowdemanded hardware realization have been proposed for soft-decision decoding in MTR basic AND, OR and XOR logic circuits. The suggested approach is explored over the two-track, two-head $\mathrm{E}^{2} \mathrm{PR} 4$ partial response magnetic recording system. The overall two-track channel detection complexity reduction of $41.9 \%$ is offered in simulation scheme, encoded by Low-Density Parity-Check (LDPC) code, serially concatenated with inner MTR. The $1.9 \mathrm{~dB}$ coding gain has been obtained, comparing to uncoded channel and assuming the AWGN noise presence.
\end{abstract}

Keywords. Soft-decision decoding; partial response; multiple-head magnetic recording systems; maximum transition run codes; constrained coding.

\section{Introduction}

The insatiable need for increasing areal recording density in hard-disc drives is likely to continue in foreseeable future. At this time, widely adopted approaches to increase areal density are increasing linear density (by reducing length of magnetic domains along the tracks) and increasing radial density (by reducing the track pitch).

The radial density increasing is followed by the multi-dimensional detection techniques utilizations. Multiple-head arrays have been developed enabling reading and writing data at the same time on multiple tracks (Barbosa 1990). Such heads can potentially provide both high density and high speed (Voois \& Cioffi 19992a, b), but they suffer from intertrack interference (ITI). This ITI is a result of a signal induced in heads due to the superposition of magnetic transitions in neighbouring tracks. Its harmful influence can be controlled until some level afterward ITI becomes one of significant factors that limit accurate channel detection, in magnetic recording systems, with highly increased radial density (Soljanin \& Georghiades 1998; Ahmed et al 2001).

Even multiple-head systems own many other excellent features, such as robustness to the reading head misalignment (Barbosa et al 1993), the potentially high overall complexity of realization is one of the reasons of their insufficient usage. In order to decrease the overall 
channel detection complexity utilization of constrained coding was suggested (Karabed et al 1999; Van Wijngaarden \& Immink 2001). Such kind of coding is used to impose restrictions on sequences of transitions, such that specific error-patterns are avoided (Altekar et al 1999). Consequently, certain minimum distance is maintained between the successive transitions, in magnetization of recording track, helping channel detector to make better estimations. Therefore, practical recording systems should employ certain type of constrained coding, and as a possible solution the maximum-transition-run (MTR) codes could be considered (Moon \& Brickner 1996).

Research of iterative decoding/detection techniques (Berrou et al 1993) for applications in magnetic recordings, have created a great deal of interest (Lynch et al 2004). Such techniques assume that decoders/detectors, operate with the soft-values (Hagenauer 1995), where sign of soft-value corresponds to the hard-decision while magnitude stands for reliability of decision. The whole decision process is known as soft-decision. Additionally, exchange of soft-values, known as an extrinsic information, is present between decoders and detectors, in such systems.

The known MTR codes are found on the hard-decision basis, producing on their output only symbols 0/1, without any other information about decision, itself. Therefore, our idea was to somehow implement soft-decision decoding in classical MTR codes, employing at same time constraints of these codes in recording systems. In this paper, we present a potential approach for the effortless MTR soft-decision decoding, accomplished with hardware low-demands mathematical approximations (Djuric 2005). We try to realize soft-decision on output of the basic MTR logic circuits, and to develop, a new one, the soft-decision MTR decoder, based on such circuits.

A brief preview on MTR codes is given in section 2, while an approach for the soft-decision decoding in MTR logic circuits is presented in section 3. Application of method over twotrack, two-head $E^{2} \mathrm{PR} 4$ recording system is given in section 4 , while simulation scenario with LDPC-MTR serial concatenation is presented in section 5. Simulation results are given in section 6 , while conclusions are provided in section 7 .

\section{Brief overview of MTR codes}

Partial response signalling is used to model communications channels with intersymbol interference (ISI), such as magnetic-recording channel (Thapar \& Patel 1987). It is known that for high order partial response model, such as the $E^{2} \mathrm{PR} 4$ channel model, ISI is so severe that the channel fail to achieve the matched-filter bound (MFB) for symbol error rate, assuming maximum-likelihood decoding. Their performance can be improved to the MFB with codes based on constrained systems, some of which may even simplify the Viterbi detectors relative to the uncoded channels (Karabed et al 1999).

MTR codes are specially designed for the $\mathrm{E}^{2} \mathrm{PR} 4$ partial response channel, where constrains the maximum number of consecutive transitions, in written magnetization sequence, increasing minimum squared Euclidean distance from 6 to 10 and providing $2.2 \mathrm{~dB}$ coding gain, when the additive white Gaussian noise (AWGN) is present (Karabed et al 1999). The MTR constraint can be described as MTR $\left(k_{1}, j\right)$ where $k_{1}$ is transition run constraint, and $j$ is the usual RLL constraint.

Hardware realization of MTR codes could be based on logic circuits (Moon \& Brickner 1996), providing a simple and low-cost realization, as it is shown in figure 1.

Logic circuits are preferred in any kind of hardware realization, because existing technology of integrated circuits provides their quite simple realization. Unfortunately, codes which can 


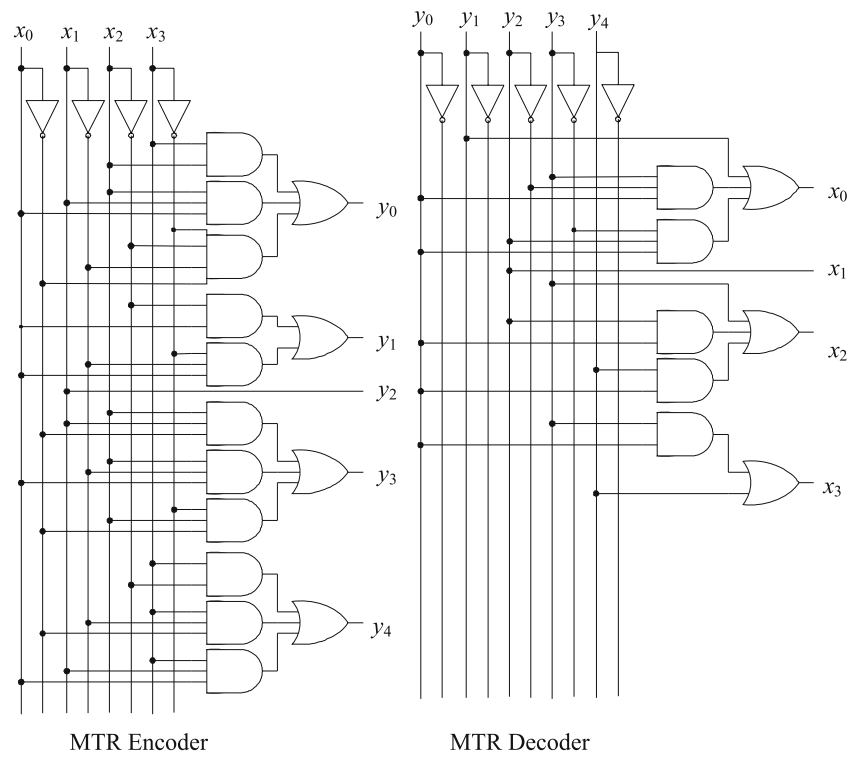

Figure 1. Rate $4 / 5\left(k_{1}=2,8\right)$ MTR code implementation.

be found on logic circuits are fated on their hard-decision base. In spite of this, the simple hardware realization, of MTR codes, was a motivating point to incorporate soft-decisions in basic logic circuits so that their output contains decision reliability as well.

\section{An approach for the soft-decisions in logic circuits}

In modern decoding techniques the soft-output value or soft-value has been widely accepted as essential information for accurate decoder decisions (Hagenauer 1995). Soft-value of the binary variable $x$ is defined using the log-likelihood ratio $(L L R)$ as:

$$
\operatorname{LLR}(x)=\log [P(x=0) / P(x=1)],
$$

In case of NOT logic, easily can be shown that $L L R$ of circuits output is:

$$
\begin{aligned}
L L R_{\text {out }}(\bar{x}) & =\log [P(\bar{x}=0) / P(\bar{x}=1)] \\
& =\log [P(x=1) / P(x=0)]=-L L R(x),
\end{aligned}
$$

which means that simple propagation of soft-values can be realized, through the NOT logic circuits. With this approach soft-value is present at the output of circuit, and thus the circuit possesses, besides the hard-decision, the decision reliability, and also it is equipped for the soft-decision process.

Same approach can be applied for the output of AND logic circuit, resulting with $L L R$ :

$$
L L R_{\text {out }}\left(x_{1} \operatorname{AND} x_{2}\right)=\log \left(e^{L L R\left(x_{1}\right)}+e^{L L R\left(x_{2}\right)}+e^{L L L\left(x_{1}\right)+L L R\left(x_{2}\right)}\right),
$$

where statistical independency of two input binary variables $x_{1}$ and $x_{2}$ have to be assumed. Unluckily, the statistical independency can not be expected in all situations, but for us it is necessary, in order to obtain some kind of useful expressions. In addition, effortless propagation of soft-values in equation (3), can not be simply accomplished, because realization of 
the $\log ()$ and $\exp ()$ functions, in digital systems, is not an easy task. Therefore, in order to simplify suggested approach we had to employ a few mathematical approximations with low hardware demands.

In case of the $L L R$ of AND logic output, the following approximation:

$$
L L R_{\text {out }}^{\text {app }}\left(x_{1} \text { AND } x_{2}\right)=\max \left[L L R\left(x_{1}\right), L L R\left(x_{2}\right)\right]
$$

simplifies hardware realization of the soft-values propagation through the circuit (Djuric \& Despotovic 2005).

$L L R$ of the OR circuit output can be expressed as:

$$
L L R_{\text {out }}\left(x_{1} \text { OR } x_{2}\right)=-\log \left(e^{-L L R\left(x_{1}\right)}+e^{-L L R\left(x_{2}\right)}+e^{-\left(L L L\left(x_{1}\right)+L L R\left(x_{2}\right)\right)}\right),
$$

where statistical independency was presumed. Straightforward hardware realization, in this case, is possible using approximation (Djuric \& Despotovic 2005):

$$
L L R_{\text {out }}^{\mathrm{app}}\left(x_{1} \text { OR } x_{2}\right)=\min \left[\operatorname{LLR}\left(x_{1}\right), \operatorname{LLR}\left(x_{2}\right)\right],
$$

Finally, assuming statistical independency as well, the $L L R$ of the XOR logic output is:

$$
L L R_{\text {out }}\left(x_{1} \text { XOR } x_{2}\right)=\log \left(\left(1+e^{L L R\left(x_{1}\right)} e^{L L R\left(x_{2}\right)}\right) /\left(e^{L L R\left(x_{1}\right)}+e^{L L R\left(x_{2}\right)}\right)\right),
$$

while simple hardware realization is possible using approximation (Hagenauer 1995):

$$
\begin{aligned}
& L L R_{\mathrm{out}}^{\mathrm{app}}\left(x_{1} \mathrm{XOR} x_{2}\right) \\
& \quad=\operatorname{sign}\left[L L R\left(x_{1}\right)\right] \cdot \operatorname{sign}\left[L L R\left(x_{2}\right)\right] \cdot \min \left[\left|L L R\left(x_{1}\right)\right|,\left|L L R\left(x_{2}\right)\right|\right] .
\end{aligned}
$$

With suggested approximations for $L L R$ implementation the soft-values can be simply spread through the basic logic circuits, preserving realization complexity of MTR decoder at the same level as in case of ordinary logic circuits utilization.

\section{Application of MTR constraints}

The MTR constraint of some patterns, in recording sequence, reflects to the E ${ }^{2} \mathrm{PR} 4$ detection trellis. Its reduction has the impact to the complexity of channel detection. Thus, the basic intention of the MTR code utilization was reduction of channel detection complexity, and also the overall complexity of simulation scheme.

\subsection{Application over one-track, one-head $E^{2} P R 4$ channel}

MTR codes are developed to prevent the $\pm[+1-1+1]$ error-event (Altekar et al 1999), in magnetic recording sequence. The result of this prevention is elimination of the 1010 and 0101 patterns from input sequence, leading to the detection trellis reduction, of one-track, one-head $E^{2}$ PR4 channel, from 16 to 14 states, as it is shown in figure 2.

As a consequence, number of states of detection trellis is reduced for $12.5 \%$. These reductions have to be reflected to the channel detection complexity. To show this, let us denote with $S_{k-1, i}$ the $i$-th state of one-track, one-head detection trellis at the moment $k-1$. Transition to the state $S_{k, j}$ is determined with input bit $x_{k} \in\{-1,+1\}$, at the moment $k$. It means that in a regular case of trellis there are two possible outgoing transitions from state and also two possible incoming transitions to the specific state. 


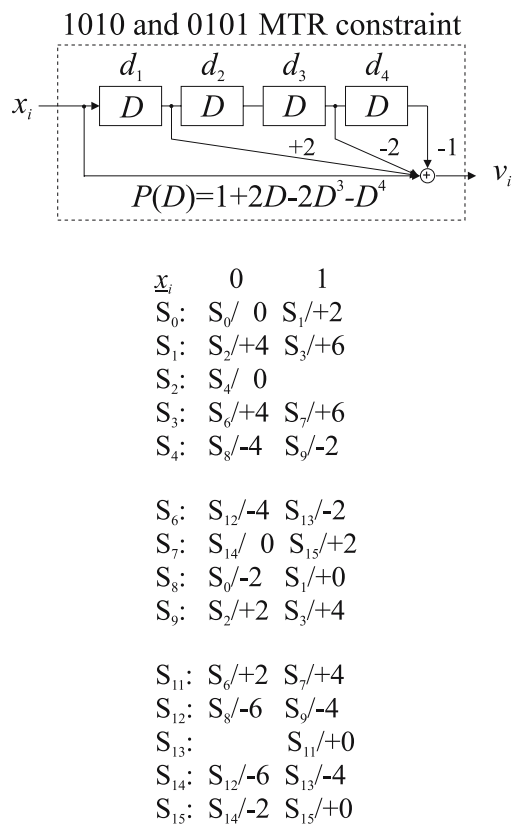

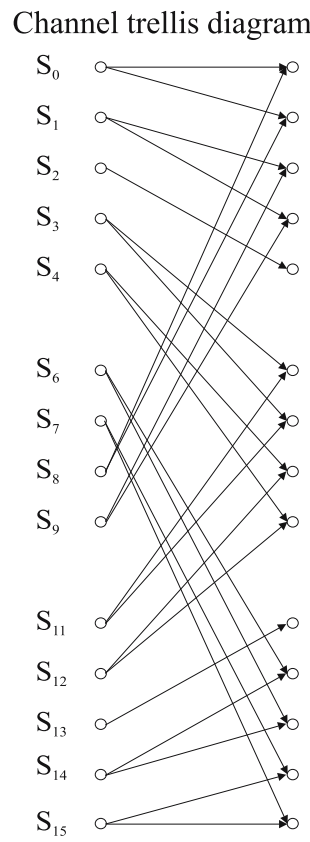

Figure 2. One-track E ${ }^{2} \mathrm{PR} 4$ channel trellis with MTR constraints.

Suppose that incoming transition to the state $S_{k, j}$ have metrics denoted with $M_{k, j}^{m}$, where $m \in\{0,1\}$ represents specific transition, and that transition denoted with $m=0$ have better metric, then difference between metrics can be defined as:

$$
\Delta_{k}=0 \cdot 5 \cdot\left[M_{k, j}^{0}-M_{k, j}^{1}\right] .
$$

It should be noticed that in one-track detection trellis, with MTR constraints, there are two states with only one incoming transition, which do not require comparison and subtraction for calculation of metric difference.

Probability that soft-output Viterbi detector (SOVA) (Hagenauer 1995), over one-track, one-head recording system, will select the best transition or the best path is:

$$
\begin{aligned}
P(\text { best path }) & =P(\text { path } 0) / \sum_{m=0}^{1} P(\text { path } m) \\
& =e^{\frac{1}{2} M_{k, j}^{0}} / \sum_{m=0}^{1} e^{\frac{1}{2} M_{k, j}^{m}}=1 /\left(1+e^{-\Delta_{k}}\right),
\end{aligned}
$$

while the reliability of that decision is:

$$
\log [P(\text { best path }) /(1-P(\text { best path }))]=-\log e^{-\Delta_{k}^{n}}=\Delta_{k} .
$$

Based on this reliability SOVA generates soft-value for estimated recorded bit as:

$$
L\left(x_{k-\tau}\right) \approx x_{k-\tau} \cdot \min _{l=0, \ldots \tau} \Delta_{k-l}
$$

where $\tau$ represents the SOVA detector decision delay. It is well known that $\min ()$, in equation (12), has to be taken only over those transitions where bits differ (Hagenauer 1995). 


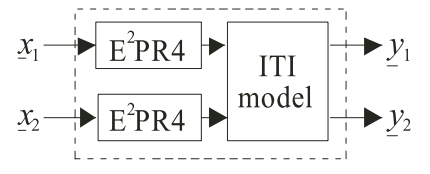

Figure 3. Two-track, two-head magnetic recording model.

In case with no constraints in detection trellis, the number of operations necessary to find the best path and reliability of that decision, for all states in moment $k$, is:

$$
N^{\text {oper. }}=16 \times(1 \text { comparison }+1 \text { subtraction })=32,
$$

according to equations (9) and (11), while in a case that the MTR constraint is present this number is:

$$
N_{\text {MTR }}^{\text {oper. }}=12 \times(1 \text { comparison }+1 \text { subtraction })=24,
$$

which corresponds to the $25 \%$ of the necessary operations decreasing and, also, of the over-all channel detection complexity reduction.

\subsection{Application over two-track, two-head $E^{2} P R 4$ channel}

In this paper, we consider a special type of multiple-head magnetic recording systems, modelled with two-track, two-head $E^{2}$ PR4 recording channel, as depicted in figure 3.

In this model, two independent tracks exist and reading heads simultaneously detects signals from both tracks. It was assumed the presence of linear and symmetrical ITI, given by the following matrix:

$$
\mathrm{A}=\left[\begin{array}{ll}
1 & \varepsilon \\
\varepsilon & 1
\end{array}\right]
$$

where $\varepsilon \in[0,1]$ represents ITI level between tracks (Soljanin \& Georghiades 1998).

The state space of the two-track detection trellis is obtained as a cross product of state spaces of two independent one-track trellises. In a case without constraints, the number of states of two-track detection trellis is $N_{\text {states }}=2^{4} \cdot 2^{4}=2^{8}=256$. Employing MTR constraints in recoding sequences along each track, some states are eliminated and the overall number of states decreases to 196 states, which is $23.4 \%$ of direct state reduction, in two-track detection trellis. Moreover, in such trellis 4 states have only one outgoing branch, the 52 states have two, while the 140 states have four outgoing branches. These valuable facts reflect to the detection complexity and simplify two-track detector functionality.

Denote with $S_{k-1, i}^{2 D}$ the $i$-th state of two-track trellis at the moment $k-1$. Transition to the state $S_{k, j}^{2 D}$ is determined with pair of input bits $\left(x_{1 k}, x_{2 k}\right)$, which are bits in corresponding tracks at the moment $k$. It means that in a regular case there are four possible outgoing transitions or branches, from specific state, and also four possible incoming transitions to state.

Assuming that incoming branches to $S_{k, j}^{2 D}$ state have metrics denoted with $M_{k, j}^{m}$, where $m \in\{0,1,2,3\}$ represents the specific branch, and that branch denoted with $m=0$ have the best metric, then differences between metrics can be defined with:

$$
\Delta_{k}^{n}=0 \cdot 5 \cdot\left[M_{k, j}^{0}-M_{k, j}^{1}\right]
$$


where $n \in\{1,2,3\}$. Probability that SOVA detector for two-track system (2H-SOVA) will select the best transition or the best path is:

$$
\begin{aligned}
P(\text { best path }) & =P(\text { path } 0) / \sum_{m=0}^{3} P(\text { path } m) \\
& =e^{\frac{1}{2} M_{k, j}^{0}} / \sum_{m=0}^{3} e^{\frac{1}{2} M_{k, j}^{m}}=1 /\left(1+\sum_{n=1}^{3} e^{-\Delta_{k}^{n}}\right),
\end{aligned}
$$

while the reliability of this decision is (Djuric \& Despotovic 2006):

$$
\begin{aligned}
\Delta_{k} & =\log [P(\text { best path }) /(1-P(\text { best path }))] \\
& =-\log \sum_{n=1}^{3} e^{-\Delta_{k}^{n}} \approx-\max _{n}\left(-\Delta_{k}^{n}\right)=\min _{n}\left|\Delta_{k}^{n}\right| .
\end{aligned}
$$

Based on best path reliability $2 \mathrm{H}-\mathrm{SOVA}$ generates soft-output values for estimated recorded bits as:

$$
\begin{aligned}
& L\left(x_{1, k-\tau}\right) \approx x_{1, k-\tau} \cdot \min _{l=0, \ldots \tau} \Delta_{k-l} \\
& L\left(x_{2, k-\tau}\right) \approx x_{2, k-\tau} \cdot \min _{l=0, \ldots \tau} \Delta_{k-l},
\end{aligned}
$$

where $\tau$ represents detector decision delay.

In a regular situation, with no constraints in detection trellis, number of operations necessary to find the best path and reliability of that decision, for all states, is:

$$
N^{\text {oper. }}=N_{\text {states }} \times 3 \text { subtraction } \times 3 \text { comparison }=1536,
$$

according to (16) and (18), while in a case that MTR constraint is present this number is:

$$
N_{\text {MTR }}^{\text {oper. }}=142 \times 3 \text { subtraction } \times 3 \text { comparison }+52 \times 1 \text { subtraction }=892,
$$

which corresponds to the $41.9 \%$ of the overall channel detection complexity reduction.

\section{LDPC-MTR concatenation over two-track, two-head $E^{2}$ PR4 channel}

The MTR constraint capabilities have been promptly recognized, such as their poor error correcting facilities. The error correction of MTR codes is looser compared to the more powerful and, in recent, mostly investigated low-density parity-check (LDPC) code (MacKay \& Neal 1997). Valid LDPC codeword are sparser in the set of all possible codeword, then those satisfying MTR constraints. Thus, LDPC have a chance to correct as many errors as it is possible.

Despite poor error correctness there were several attempts to utilize MTR constraints in conjunction with some powerful error correcting code (ECC) (Todd \& Cruz 2004; Kanaoka \& Morita 2006). Those approaches are based on employment of MTR as an outer and ECC as an inner encoding code. 


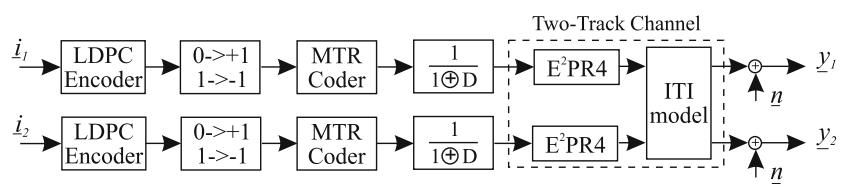

Figure 4. In-track LDPC-MTR coding over two-track channel.

In this paper, we consider the reverse order of encoders, suggesting employment of the ECC as an outer encoding code, in a simple concatenation with inner MTR code (Djuric \& Despotovic 2006). With such simulation scenario, MTR is near the recording channel and its constraints can be fully utilized for reduction of channel detection trellis. Also, soft-values are exchanged between channel detector and outer ECC, without addition of any new model and without increasing simulation scheme complexity.

Our intention was to investigate the behaviour of the soft-decision versus hard-decision MTR decoding, in a strong error correcting system, and thus the LDPC-MTR was the natural choice. At this moment our target was not implementation of any specially designed or matched codes, and therefore the LDPC-MTR concatenation has been performed using rate $R=0.96 \mathrm{LDPC}$, as an outer, and rate 4/5 MTR $\left(k_{1}=2,8\right)$ (Moon \& Brickner 1996), as an inner encoding code. Each track was independently encoded as shown in figure 4.

LDPC code of length $N=4732$ with $M=169$ parity bits, and with column-weight 3 , is based on the Kirkman triple systems (Vasic et al 2002). The overall code rate is $R=0.771$.

It was assumed that in-track read-back signals are distorted with additive white Gaussian (AWGN) noise- $n$ and that the signal-to-noise ratio (SNR) is defined as:

$$
\mathrm{SNR}=10 \log \left(E_{b} / N_{o}\right)=10 \log \left(E_{c} / 2 R \sigma^{2}\right),
$$

where $E_{c}=R E_{b}$ is symbol bit energy at channel output, $N_{o}$ is one-sided power spectral density and $\sigma^{2}$ is noise variance.

The two-track channel detection was performed with $2 \mathrm{H}$-SOVA detector, with perfect ITI estimation and 20 symbols detection window. The main advantage of employing SOVA detector, however, is a reduction in complexity by about $50 \%$ relative to that of an a posteriori probability (APP) detector (Robertson et al 1995). In addition, the SOVA decoder, unlike APP detector, does not require knowledge of channel noise variance (Cong et al 1999), which makes it even more attractive.

Assuming that $y_{k i}$ is received symbol at time instant $i$, in track $k$, the 2H-SOVA branch distance between $\left(y_{1 i}, y_{2 i}\right)$ and noiseless trellis transition label $\left(v_{1 i}, v_{2 i}\right)$, is:

$$
\left[y_{1 i}-\left(v_{1 i}+\varepsilon v_{2 i}\right)\right]^{2}+\left[y_{2 i}-\left(\varepsilon v_{1 i}+v_{2 i}\right)\right]^{2},
$$

where $\left(u_{1 i}, u_{2 i}\right)$ is corresponding information bits label for ITI-based trellis and $\varepsilon$ represents the ITI level between tracks (Soljanin \& Georghiades 1998; Djuric \& Despotovic 2006; Ghrayeb \& Huang 2005).

Moreover, in order to decrease the overall complexity it was considered the case when 2H-SOVA only forwards soft-output information to the in-track message-passing modules, through the MTR decoders, and without exchange between them, as shown in figure 5.

In all simulations message-passing algorithm operates through only one iteration, and when finishes with processing, a decision about the LDPC code word is made.

In order to emphasize the soft-decision decoding of MTR codes we additionally simulate situation when hard-decision of MTR is utilized. In these circumstances the soft-values from SOVA have been first used to produce the hard-decisions of sequence which is then forwarded 


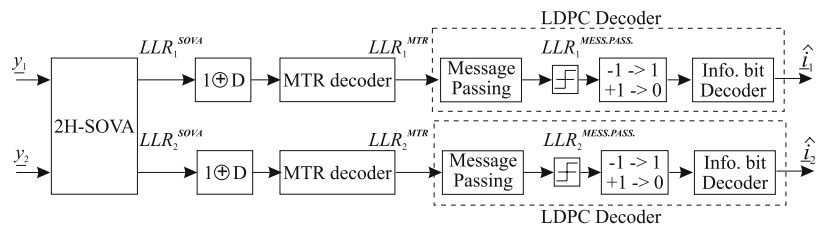

Figure 5. Decoding in two-track, two-head system.

to the classical MTR decoder. Later, hard-decision output of the MTR decoder is forwarded as its soft-values to the message-passing. In that moment, message-passing algorithm receives sequence with only $-1 /+1$ information, where the reliability of bits decisions are already predetermined and fixed. This is not desirable situation to the message-passing, but it was the only method to incorporate classical hard-decision of MTR codes, in LDPC-MTR concatenation, so that MTR acts as an inner encoding code.

\section{Simulation results}

Simulation results for two-track, two-head system encoded by LDPC-MTR concatenation and decoded with usual hard-decision and the new one soft-decision decoding, of MTR codes, are shown in figure 6.

Depicted simulation results, at the first, demonstrate the well known two-head detector robustness on ITI presence, over range of ITI values $0 \leq \varepsilon \leq 0 \cdot 293$, where additional coding gain has been obtained, comparing with no ITI case $(\varepsilon=0)$ (Soljanin \& Georghiades 1998; Djuric \& Despotovic 2006; Djuric \& Despotovic 2003). This gain is present despite which approach of the in-track MTR decoding was used. After this level ITI influence can not be eliminated, and decoding results are highly inferior.

Employing LDPC-MTR concatenation with soft-decision MTR decoder, nearly $1.9 \mathrm{~dB}$ coding gain has been obtained for ITI levels $\varepsilon=0$ and $\varepsilon=0 \cdot 2$, compared to the same level

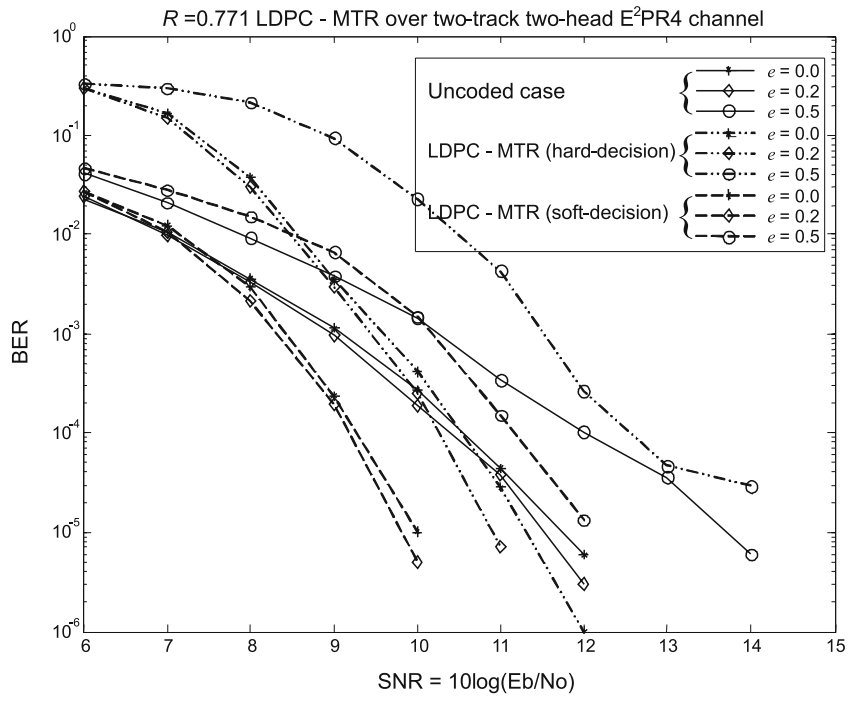

Figure 6. LDPC-MTR code over two-track, two-head E2 PR4 channel model. 
of ITI in uncoded case, and at the BER $=10^{-5}$. In this situation for ITI level of $\varepsilon=0 \cdot 5$, also, $1.9 \mathrm{~dB}$ coding gain exists.

When LDPC-MTR concatenation with usual hard-decision MTR decoding, only $0.4 \mathrm{~dB}$ coding gain for ITI level $\varepsilon=0$ and $0.6 \mathrm{~dB}$ gain for $\varepsilon=0.2$ are obtained, for the BER $=10^{-5}$. In this scenario for ITI level of $\varepsilon=0 \cdot 5$, the worst result exists in comparison with uncoded case.

Comparing the cases when the soft-decision and hard-decision MTR decoding are implemented, soft-decision decoding is superior for nearly $1.2 \mathrm{~dB}$ at ITI level $\varepsilon=0$ and $1.1 \mathrm{~dB}$ at ITI level $\varepsilon=0 \cdot 2$, then the hard-decision approach for the BER $=10^{-5}$.

The reasons lie in information which arrives to the message-passing decoder. Information based on hard-decisions does not contain the valuable reliability about decisions and thus it can not be properly utilized in message-passing decoder. On the other side, the MTR soft-decision decoding forward decisions and their reliabilities, which are additional helpful information for the message-passing decoder.

\section{Conclusion}

In this paper, an approach for the soft-decision decoding of MTR codes has been considered. It is shown that effortless propagation of soft-output information can be obtained, utilizing $L L R$ facilities in basic logic circuits. The low-demanded mathematical approximations were used to implement soft-decision decoding in low-level MTR circuits.

In a framework of the two-track, two-head $E^{2} \mathrm{PR} 4$ magnetic recording system, utilization of the soft-decision MTR decoding resulted in nearly $1.9 \mathrm{~dB}$ coding gain, compared to the uncoded case, for all ITI levels, at the BER $=10^{-5}$. Moreover, the soft-decision approach is superior for $1.2 \mathrm{~dB}$ at ITI level $\varepsilon=0$ and $1.1 \mathrm{~dB}$ at ITI level $\varepsilon=0 \cdot 2$, then the hard-decision method. Furthermore, about $23 \%$ of detection trellis states reduction and $41.9 \%$ of overall channel detection complexity reduction is observed in that case.

Combining simulation results for soft-decision MTR decoding with $41.9 \%$ of the overall two-track detection complexity reduction, LDPC-MTR concatenation demonstrate potentials to become important part in multiple-head magnetic recording systems.

\section{List of symbols}

$L L R \quad$ the log-likelihood ratio,

$L L R_{\text {out }}$ the log-likelihood ratio of the circuit output,

$L L R_{\text {out }}^{\text {app }}$ approximation of the log-likelihood ratio of the circuit output,

$x_{1}, x_{2} \quad$ binary variables,

$S_{k-1, i} \quad$ the $i$-th state of one-track one-head detection trellis at moment $k$-1,

$S_{k, j} \quad$ the $j$-th state of one-track one-head detection trellis at moment $k$,

$M_{k, j}^{m} \quad$ metrics of the incoming transition to the state $S_{k, j}$,

$\Delta_{k} \quad$ difference between metrics of one-track trellis in moment $k$,

$N^{\text {oper. }} \quad$ number of operations necessary to find the best path and reliability,

$N_{\text {MTR }}^{\text {oper. }} \quad$ number of operations necessary to find the best path and reliability with MTR,

A ITI matrix, 


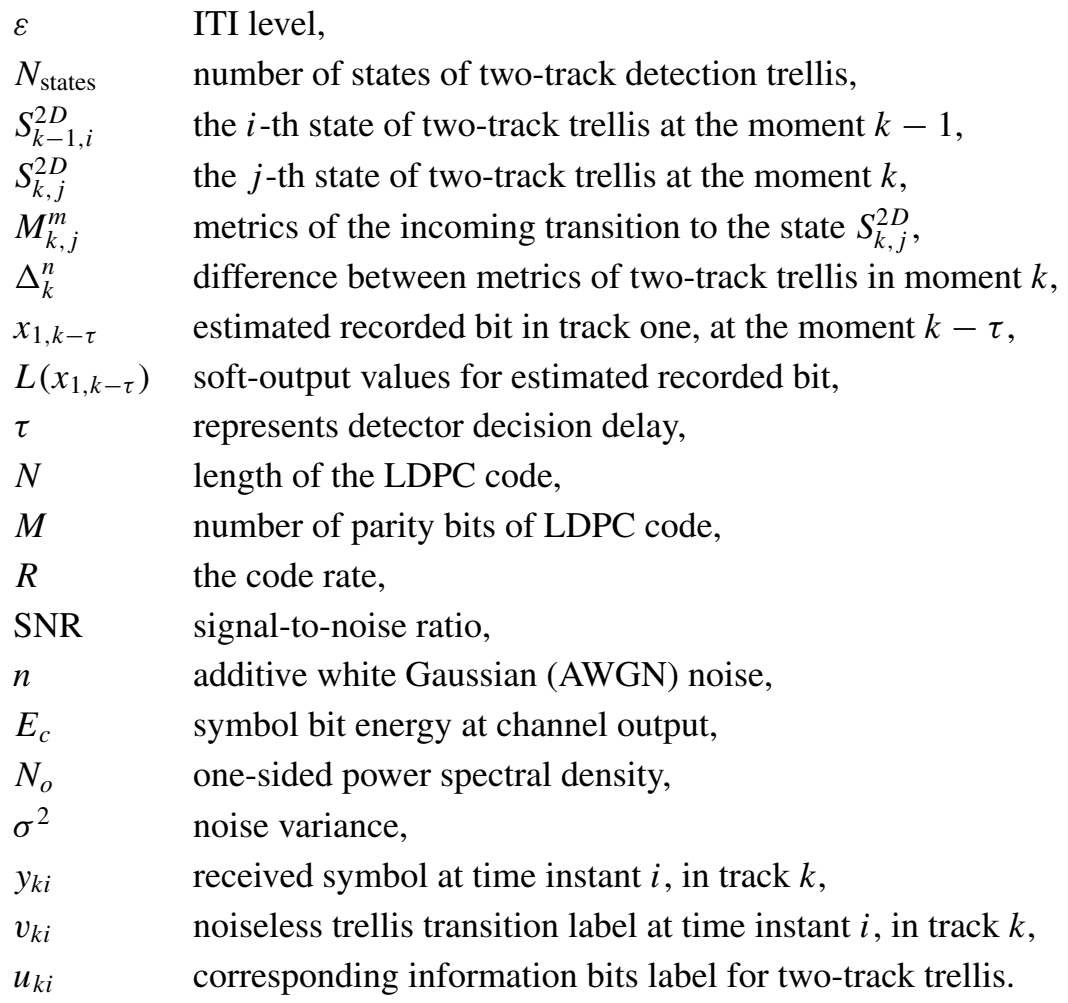

\section{References}

Ahmed M Z, Donnely T, Davey P J, Clegg W W 2001 Increased areal density using a two-dimensional approach. IEEE Trans. Mag. 37: 1896-1898

Altekar S A, Berggren M, Moision B M, Siegel P H, Wolf J K 1999 Error-event characterization on partial-response channels. IEEE Trans. Inform. Theory 45(1): 241-247

Barbosa L C 1990, Simultaneous detection of readback signals from interfering magnetic recording tracks using array heads. IEEE Trans. Mag. 26(5): 2163-2165

Barbosa L C, MacDonald C E, Wolf J K 1993 Array heads for estimating track misregistration. IEEE Trans. Mag. 29: 4033-4035

Berrou C, Glavieux A, Thitimajshima P 1993, Near Shannon limit error-correcting coding and decoding: Turbo codes, in the IEEE Int. Conf. Communications (ICC 1993), Conf. Record, vol. 2, Geneva, Switzerland 1064-1070

Cong L, Xiaofu W, Xiaoxin Y 1999 On SOVA for nonbinary codes. IEEE Commun. Lett. 3(12): 335-337

Djuric N 2005, Iterative decoding of LDPC - MTR code concatenation over partial response magnetic recording channels, $P S U$-UNS International Conference on Engineering and Environment - ICEE2005, Novi Sad 19-21 May

Djuric N, Despotovic M 2003, Distance analysis for $E^{2}$ PR4 two-track, two-head magnetic recording channel, Proceedings of XI conference TELFOR, Beograd, Serbia

Djuric N, Despotovic M 2005, Soft-output decoding approach of maximum transition run codes, The International Conference on "Computer as a tool", EUROCON 2005 Proceeding, Belgrade, 22-24 November, 490-493 
Djuric N, Despotovic M 2006, Soft-output decoding in multiple-head MTR encoded magnetic recording systems. In Proc. IEEE Int. Conf. Communications (ICC 2006), Istanbul, Turkey 11-15 June

Ghrayeb A, Huang C X 2005 Improvements in SOVA-based decoding for turbo-coded storage channels. IEEE Trans. Mag. 41(12): 4435-4442

Hagenauer J 1995 Source-controlled channel decoding. IEEE Trans. Comm. 43(9): 2449-2457

Kanaoka T, Morita T 2006 Structured LDPC codes with reversed MTR/ECC for magnetic recording channels. IEEE Trans. Mag. 42(10): 2561-2563

Karabed R, Siegel P H, Soljanin E 1999 Constrained coding for binary channels with high intersymbol interference. IEEE Trans. Inform. Theory 45(6): 1777-1797

Lynch R, Kurtas E M, Kuznetsov A, Yeo E, Nikolic B 2004 The search for a practical iterative detector for magnetic recording. IEEE Trans. Mag. 40(1): 213-218

MacKay D J C, Neal R 1997 Near Shannon limit performance of low density parity check codes. IEE Electron. Lett. 33: 457-458

Moon J, Brickner B 1996 Maximum transition run codes for data storage systems. IEEE Trans. Mag. 32(5): 3992-3992

Robertson P, Villebrun E, Hoeher P 1995 A comparison of optimal and sub-optimal MAP decoding algorithms operating in the log domain, in. Proc. IEEE Int. Conf. Communications (ICC 1995) $1009-1013$

Soljanin E, Georghiades C N 1998 Multihead detection for multitrack recording channels. IEEE Trans. Inform. Theory 44(7): 2988-2997

Thapar H K, Patel A M 1987 A class of partial response systems for increasing storage density in magnetic recording. IEEE Trans. Mag. vol. MAG-25: 3666-3668

Todd R M, Cruz R 2004 Enforcing maximum-transition-run code constraints and low-density paritycheck decoding. IEEE Trans. Mag. 40(6): 3566-3571

Van Wijngaarden A J, Immink K A S 2001 Maximum runlength limited codes with error control capabilities. IEEE J. Selec. Areas Commun. 19: 602-611

Vasic B, Kurtas E, Kuznetsov A 2002 Kirkman systems and their application in perpendicular magnetic recording. IEEE Trans. Mag. 38(4) Part 1: 1705-1710

Voois P A, Cioffi J M 1992a Multichannel digital magnetic recording, in. Proc. 1992 IEEE Int. Conf. Communications (ICC'92) (Chichago, IL, June 1992) 305.4·1-305.4.6

Voois P A, Cioffi J M 1992b Achievable radial information densities in magnetic recording systems, in. Proc. 1992 IEEE Global Telecommunications Conf. (GLOBECOM '92) (Orlando, FL, Dec. 1992) 1067-1071 